\title{
Standardization of pathologic evaluation and reporting of postneoadjuvant specimens in clinical trials of breast cancer: recommendations from an international working group
}

Elena Provenzano ${ }^{1}$, Veerle Bossuyt ${ }^{2}$, Giuseppe Viale ${ }^{3}$, David Cameron ${ }^{4}$, Sunil Badve ${ }^{5}$, Carsten Denkert ${ }^{6}$, Gaëtan MacGrogan ${ }^{7}$, Frédérique Penault-Llorca ${ }^{8}$, Judy Boughey ${ }^{9}$, Giuseppe Curigliano ${ }^{10}$, J Michael Dixon ${ }^{11}$, Laura Esserman ${ }^{12}$, Gerd Fastner ${ }^{13}$, Thorsten Kuehn $^{14}$, Florentia Peintinger ${ }^{15,16}$, Gunter von Minckwitz ${ }^{17}$, Julia White ${ }^{18}$, Wei Yang ${ }^{19}$ and W Fraser Symmans ${ }^{20}$ on behalf of the Residual Disease Characterization Working Group of the Breast International Group-North American Breast Cancer Group (BIG-NABCG) collaboration

${ }^{1}$ Department of Histopathology and NIH Cambridge Biomedical Research Centre, Addenbrooke's Hospital, Cambridge, UK; ${ }^{2}$ Department of Pathology, Yale University, New Haven, CT, USA; ${ }^{3}$ Department of Pathology, European Institute of Oncology and University of Milan, Milan, Italy; ${ }^{4}$ Edinburgh Cancer Research UK Centre, University of Edinburgh, Edinburgh, UK; ${ }^{5}$ Department of Pathology and Laboratory Medicine, Indiana University School of Medicine, Indianapolis, IN, USA; ${ }^{6}$ Institute of Pathology, Charité Hospital, Berlin, Germany; ${ }^{7}$ Department of Biopathology, Institut Bergonié, Bordeaux, France; ${ }^{8}$ Department of Pathology, Centre Jean Perrin and Université d'Auvergne, Clermont-Ferrand, France; ${ }^{9}$ Division of Subspecialty General Surgery, Mayo Clinic, Rochester, MN, USA; ${ }^{10}$ Early Drug Development for Innovative Therapies Division, European Institute of Oncology, Milan, Italy; ${ }^{11}$ Edinburgh Breast Unit, Western General Hospital, Edinburgh, UK; ${ }^{12}$ Carol Franc Buck Breast Care Center, University of California, San Francisco, CA, USA; ${ }^{13}$ Department of Radiotherapy and Radiation Oncology, Landeskrankenhaus, Paracelsus Medical University, Salzburg, Austria; ${ }^{14}$ Department of Gynecology and Obstetrics, Interdisciplinary Breast Center, Klinikum Esslingen, Esslingen, Germany; ${ }^{15}$ Institute of Pathology, Medical University of Graz, Graz, Austria; ${ }^{16}$ Department of Gynecology, General Hospital Leoben, Leoben, Austria; ${ }^{17}$ German Breast Group, Neu-Isenburg, and Department of Gynecology and Obstetrics, University Women's Hospital, Frankfurt, Germany; ${ }^{8}$ Department of Radiation Oncology, Ohio State University, Columbus, OH, USA; ${ }^{19}$ Department of Diagnostic Radiology, The University of Texas MD Anderson Cancer Center, Houston, TX, USA and ${ }^{20}$ Department of Pathology, The University of Texas MD Anderson Cancer Center, Houston, TX, USA

Neoadjuvant systemic therapy is being used increasingly in the treatment of early-stage breast cancer. Response, in the form of pathological complete response, is a validated and evaluable surrogate end point of survival after neoadjuvant therapy. Thus, pathological complete response has become a primary end point for clinical trials. However, there is a current lack of uniformity in the definition of pathological complete response. A review of standard operating procedures used by 28 major neoadjuvant breast cancer trials and/or 25 sites involved in such trials identified marked variability in specimen handling and histologic reporting. An international working group was convened to develop practical recommendations for the pathologic assessment of residual disease in neoadjuvant clinical trials of breast cancer and information expected from pathology reports. Systematic sampling of areas identified by informed mapping of the specimen and close correlation with radiological findings is preferable to overly exhaustive sampling, and permits taking tissue samples for

Correspondence: Dr E Provenzano, MBBS, PhD, FRCPA, Box 235, Addenbrooke’s Hospital, Hills Road, Cambridge CB2 0QQ, UK.

E-mail: elena.provenzano@addenbrookes.nhs.uk

Received 9 December 2014; accepted 9 March 2015; published online 24 July 2015 


\begin{abstract}
translational research. Controversial areas are discussed, including measurement of lesion size, reporting of lymphovascular space invasion and the presence of isolated tumor cells in lymph nodes after neoadjuvant therapy, and retesting of markers after treatment. If there has been a pathological complete response, this must be clearly stated, and the presence/absence of residual ductal carcinoma in situ must be described. When there is residual invasive carcinoma, a comment must be made as to the presence/absence of chemotherapy effect in the breast and lymph nodes. The Residual Cancer Burden is the preferred method for quantifying residual disease in neoadjuvant clinical trials in breast cancer; other methods can be included per trial protocols and regional preference. Posttreatment tumor staging using the Tumor-Node-Metastasis system should be included. These recommendations for standardized pathological evaluation and reporting of neoadjuvant breast cancer specimens should improve prognostication for individual patients and allow comparison of treatment outcomes within and across clinical trials.
\end{abstract}

Modern Pathology (2015) 28, 1185-1201; doi:10.1038/modpathol.2015.74; published online 24 July 2015

Neoadjuvant systemic therapy is being increasingly used in the treatment of early-stage breast cancer. Response, in the form of pathological complete response, is being put forward as an evaluable end point for determining the efficacy of new agents in neoadjuvant clinical trials ${ }^{1}$ and is an excellent prognostic indicator. ${ }^{2}$ Data are also emerging on the frequency of regional recurrence based on the presence of residual disease in both breast and lymph nodes. ${ }^{3}$ However, accurate evaluation of the original tumor bed depends on correct localization and sampling of the tumor bed. Therefore, gross pathologic methods are the single greatest determinant for accurate definition of pathological complete response or residual disease. This not only alters the end point, but could increasingly affect decisions regarding the need for further local-regional or systemic therapy, if based on the extent of residual disease. ${ }^{3}$ Therefore, a standard approach to the evaluation of the postneoadjuvant systemic therapy surgical specimen is essential.

Several classification systems have been developed for the assessment of pathologic response to neoadjuvant systemic therapy; these have been reviewed elsewhere. ${ }^{4-11}$ Although, collectively, they have their advantages and disadvantages, most have been validated as correlating with outcome (overall survival, event-free survival, and/or distant relapsefree survival). ${ }^{6,10,12-16}$ However, different staging systems yield different estimates of future risk. ${ }^{17}$ The Residual Cancer Burden is an online tool for the quantification of residual disease that is simple to apply, reproducible, and has been clinically validated with long-term follow-up data. ${ }^{10,18,19}$

Moreover, novel classification systems are continually being developed, for example, those that incorporate biomarkers in addition to traditional histologic prognostic variables, such as the Residual Proliferative Cancer Burden that combines Residual Cancer Burden with posttreatment Ki67 index. ${ }^{20}$ There are also combined clinical and pathological systems that take into account pretreatment information such as clinical stage as well as posttreatment pathology findings, for example, the 'clinical-pathologic stage-estrogen/grade’ staging system. ${ }^{21}$ These approaches also show promise as future means to predict outcome by combining additional clinical or biological information with Residual Cancer Burden or American Joint Committee on Cancer stage after treatment.

National guidelines have been developed for histopathologic assessment of breast cancer specimens in individual countries/regions, including Australasia, ${ }^{22}$ Belgium, ${ }^{23}$ Germany, ${ }^{24}$ the United Kingdom (now being updated), ${ }^{25}$ The Netherlands, ${ }^{26}$ and the United States. ${ }^{27}$ These vary in their approach to evaluating the postneoadjuvant specimen.

Frequently, neoadjuvant systemic therapy will be administered in the setting of a clinical trial. Pathologists must be involved at an early stage in trial development so that specimen handling, reporting, and tissue collection is specified. ${ }^{28}$ Currently, in many multicenter neoadjuvant systemic therapy trials, the surgical specimens are reported by the treating hospital without even minimum guidelines for specimen handling or centralized review to ensure validity and reproducibility of results. A central review of histopathology reports within the neo-tAnGo trial, a UK-based multicenter randomized neoadjuvant chemotherapy trial in early breast cancer, revealed huge variation in handling and reporting of neoadjuvant specimens between centers. ${ }^{29}$ In the I-SPY 1 trial, the pathological complete response rate fell by almost $10 \%$ among pathologists at 9 centers after they were trained on how to use the Residual Cancer Burden tool (Laura Esserman, personal communication, 2 August 2013). In a French multicenter study that used the Chevallier system, ${ }^{30}$ the pathological complete response rate in one arm of the study fell from 16 to $8 \%$ following central pathology review of slides. ${ }^{31}$

Finally, the definition of pathological complete response has not been uniform, making reporting and interpretation of data challenging. ${ }^{5,32}$ The frequency of use of different definitions of pathological complete response in major neoadjuvant clinical trials is illustrated in Figure 1. These different definitions of pathological complete response can change the apparent survival benefit associated with pathological complete response, 


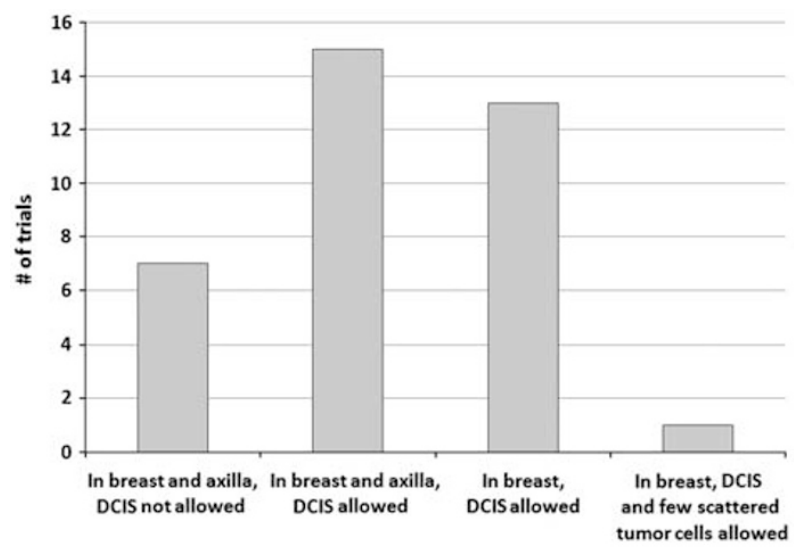

Figure 1 Use of different definitions ${ }^{\mathrm{a}}$ of pathological complete response in major neoadjuvant breast cancer clinical trials. Trials included in graphic above: first bar: GeparDuo, GeparTrio, GeparQuattro, GeparQuinto, GeparSixto, GeparSepto, NEOCENT; second bar: ABCSG 32, ACOSOG-Z1031, ACOSOG-Z1071, ARTemis, CHERLOB, CNIO-BR-01 2010, I-SPY 2, MDACC 2012-0167, neo-tAnG0, neo-TN (used Neoadjuvant Response Index), NEOZOTAC, NOAH, REMAGUS 02, S0800, TECHNO; third bar: ACOSOG-Z1041, AGO1, CALGB-40601, CALGB-40603, ECTO, GEICAM/2006-14, Neo-ALTTO, Neo-Sphere, NSABP-B-18, NSABP-B-27, NSABP-B-40, NSABP-B-41, S0012; fourth bar: EORTC-10994. ${ }^{a}$ Definition used in the primary end point or, where pathological complete response was not the primary end point, in the secondary end point.

depending upon which definition is used. (Figure 2). 2,10,15,32,33 There is general consensus that residual disease in the axillary lymph nodes indicates a worse prognosis, even when there has been a pathological complete response in the breast, and hence the definition of pathological complete response should include absence of disease in both the breast and axillary lymph nodes. . $^{2,3,17,32,34-40}$

A more contentious issue is whether the presence of residual ductal carcinoma in situ (DCIS) in the absence of residual invasive disease should be included or excluded from pathological complete response. ${ }^{32,33}$ The US Food and Drug Administration-led pooled analysis of 12 neoadjuvant randomized trials with long-term follow-up undertaken by the Collaborative Trials in Neoadjuvant Breast Cancer (CTNeoBC) found similar event-free survival and overall survival in patients without residual invasive carcinoma regardless of the presence/absence of residual DCIS. ${ }^{2}$ However, in a different statistical approach, a pooled analysis of the seven prospective neoadjuvant systemic therapy clinical trials by the German and Austrian Breast Groups demonstrated significantly worse event-free survival for patients with ypTisypNo compared with patients who were ypT0ypNo. There was no significant difference in overall survival ${ }^{32}$ (Figure 2). An analysis of a smaller cohort of patients treated at the MD Anderson Cancer Center, however, showed no difference in survival between patients with ypTOypNo and ypTisypNo (Figure 2). ${ }^{33}$ It is conceivable that an internationally uniform procedure for handling and reporting on postneoadjuvant systemic therapy specimens would eventually resolve this issue.

Overall, the US Food and Drug Administrationsupported pooled analysis was not able to validate differences in pathological complete response rate as a surrogate end point for difference in event-free survival from the neoadjuvant clinical trials included in the analysis. However, it did point to substantial improvements in survival in individuals with pathological complete response and supported standardization of the definition of pathological complete response, proposing it should be defined as either ypTo/isypNo or ypT0ypNo in future trials. ${ }^{2}$

\section{Materials and methods}

Given the lack of consensus regarding the pathological assessment of postneoadjuvant systemic therapy breast cancer specimens in clinical trials, an international working group of pathologists, radiologists, surgeons, medical and radiation oncologists, and gynecologists was convened by the BIG-NABCG collaboration. Members were nominated by BIG-NABCG leadership and the working group co-chairs, as well as by sites responding to the collection of standard operating procedures described below. Members represented an array of disciplines and countries.

First, to gauge existing variability in approaches to postneoadjuvant systemic therapy pathologic assessment, we collected standard operating procedures from neoadjuvant breast cancer trials and from sites participating in such trials. ClinicalTrials.gov was searched for mainly academic, phase II or III neoadjuvant trials activated since 2005, with a planned recruitment of at least 100 patients. Earlier trials were included if they were one of the trials included in the US Food and Drug Administration pooled analysis noted above, or otherwise were major trials (e.g., >1000 patients). Standard operating procedures were requested of 48 trials, both from the leaders of the trials themselves (trial standard operating procedures) and, where leaders responded, the sites involved in those trials (site-specific standard operating procedures). Information from the standard operating procedures was abstracted into categories of 'extent of sampling,' 'quantification/size/grading/cellularity,' 'lymph node evaluation,' 'retesting of markers,' and 'other information.' The abstracted information was then compared and contrasted.

The working group convened on seven teleconferences and three smaller planning calls, exchanged emails, and went through several rounds of comments, resulting in the development of practical recommendations for a minimum, essential set of components that should be included in the pathologic evaluation and reporting of postneoadjuvant systemic therapy breast cancer specimens. The working group has also written a companion paper intended for a 


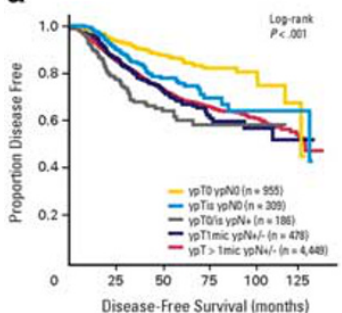

d

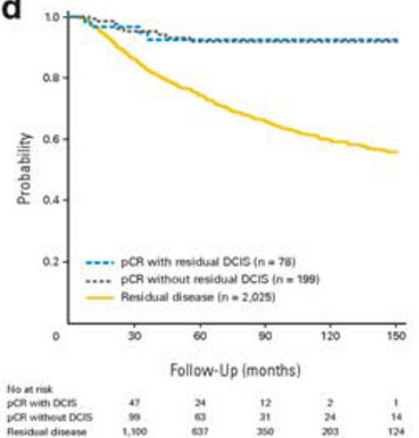

b
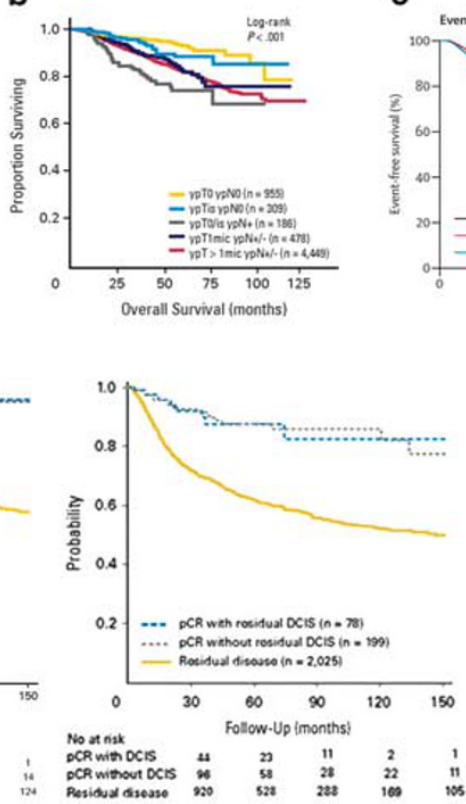

C
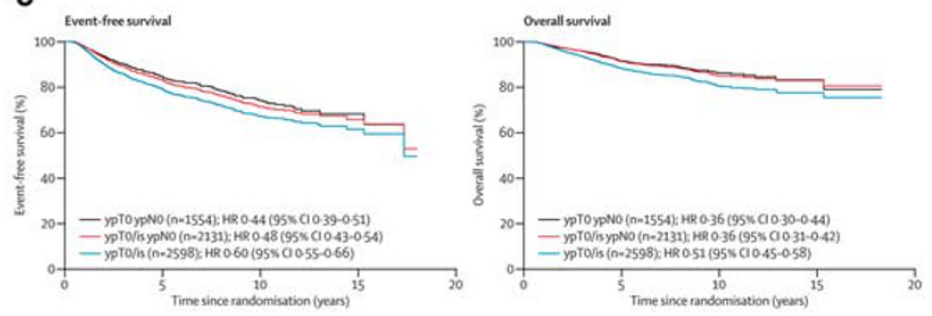

e
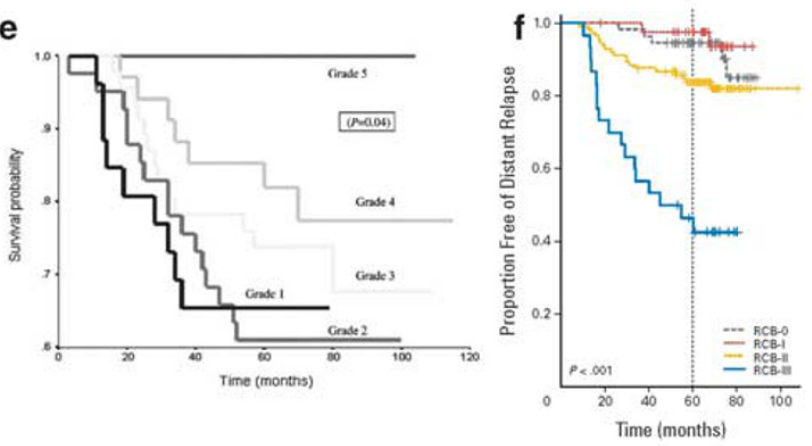

Figure 2 Survival curves showing impact of different definitions of pathological complete response on survival. (a) Data from the German Breast Group and AGO-B trials pooled analysis showing reduced disease-free survival for patients with ypTisypN0 vs ypT0ypN0. Patients who had residual nodal disease despite absence of invasive cancer in the breast (ypT0/isypN+) had the poorest disease-free survival. ${ }^{32}$ (b) Results from the same study showing no significant difference in overall survival between ypT0ypNo and ypTisypNo. The ypT0/isypN+ has a significantly worse overall survival compared with ypT0/isypNo. (c) In the CTNeoBC pooled analysis, ${ }^{2}$ ypT0pNo and ypT0/isypNo were more strongly associated with improved event-free survival and overall survival than ypT0/is alone. Moreover, ypT0ypN0 and ypT0/ isypNo were similar in their associations to event-free survival and overall survival. (d) MD Anderson study showing 5- and 10-year overall survival (left) and disease-free survival (right) rates were identical for the patients with pathological complete response vs pathological complete response+DCIS. ${ }^{33}$ (e) Categories of reduction in cellularity in the Miller-Payne system correlate with disease-free survival. ${ }^{15}$ (f) Residual Cancer Burden score is an independent variable that predicts likelihood of relapse. Minimal residual disease (RCBI) carries the same prognosis as pathological complete response. ${ }^{10}$ (a and b) Reprinted with permission. (C) 2012 American Society of Clinical Oncology. All rights reserved. von Minckwitz et al. ${ }^{32}$ (c) Reprinted from Cortazar et al. ${ }^{2}$ @ 2014 with permission from Elsevier. (d) Reprinted with permission. @ 2007 American Society of Clinical Oncology. All rights reserved. Mazouni et al. ${ }^{33}$ (e) Reprinted from Ogston et al. ${ }^{15}$ () 2003, with permission from Elsevier. (f) Reprinted with permission. ( 2007 American Society of Clinical Oncology. All rights reserved. Symmans et al. ${ }^{10}$

more multidisciplinary audience, explaining how a standardized approach would benefit the entire medical team and summarizing the more detailed recommendations provided below. ${ }^{41}$

\section{Results}

Standard operating procedures were collected from 28 trials and 25 sites (Supplementary Information 1). Substantial variability of practice was found in all stages of histological evaluation of both breast and nodal neoadjuvant specimens: extent of sampling (ranging from 4 to 40 blocks, depending on presence/ absence of a macroscopic identifiable lesion and on tumor size), thickness of primary-tumor sectioning (ranging from 2 to $10 \mathrm{~mm}$ ), the routine performance of immunohistochemical staining when no tumor was identified on hematoxylin and eosin, how amount of residual tumor was measured and documented, and whether or not a formal system was used to grade response and, if so, which system was used. For small specimens, most sites submitted the entire specimen. Only 6 of 20 sites that discussed retesting of markers in their response noted they retested markers routinely. Of note, several sites emphasized a need for standardization of the pathologic assessment of postneoadjuvant systemic therapy specimens, within practicable limits. Further details are provided in Supplementary Information 1.

\section{Recommendations}

The working group's practical suggestions are detailed below.

\section{Pretreatment Assessments}

Initial diagnosis on core biopsy of the breast Percutaneous image-guided core needle biopsy is strongly recommended, and must be adequate for an unequivocal diagnosis of invasive breast carcinoma. Caution must be used if imaging or core needle biopsy findings suggest that a significant portion of the lesion may represent in situ disease, or if there is only a limited amount of invasive carcinoma represented in the core. In these cases, repeat core needle 
biopsy or surgery for accurate diagnosis, rather than neoadjuvant systemic therapy, may be indicated. Histologic type, tumor grade, estrogen receptor (ER), progesterone receptor (PR), and HER2 status, as well as any other parameters used to select for neoadjuvant systemic therapy (e.g., Ki67, multigene assays) should be evaluated on the core needle biopsy.

Several systems for grading tumor response to treatment require comparison of cellularity with the pretreatment biopsy, such as the Miller-Payne,

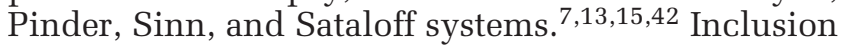
of an estimate of tumor cellularity in the core needle biopsy is of value if these systems will be used to grade response in the excision specimen.

Consideration should be given to dedicated baseline cores for research, either at the time of diagnostic biopsy or as a separate designated biopsy procedure. ${ }^{43}$ Research cores should be in addition to those required for diagnosis and should be preserved in order to best meet the research need. Touch preparations or frozen sections can be used to confirm the presence of malignant cells in the dedicated research cores before freezing or immersion into a dedicated solution. If using Optimum Cutting Temperature freezing media, one tissue core can be embedded per block. In some cases, formalinfixed cores can be reembedded as a research block after reporting. Some trials also require 'on-treatment' research core biopsies at subsequent time points (e.g., after the first cycle or at mid-course).

\section{Evaluation of the axilla before treatment}

Routine axillary ultrasound is recommended to assess the axillary lymph nodes, with fine needle aspiration or core needle biopsy of morphologically abnormal lymph nodes. Thus, sentinel lymph node biopsy before neoadjuvant treatment should be limited to cases where the pretherapeutic lymph node status is required for systemic or local treatment decisions. ${ }^{44}$ Pretreatment sentinel lymph node biopsy precludes assessment of nodal response to neoadjuvant systemic therapy, and invalidates American Joint Committee on Cancer yp stage and calculation of the Residual Cancer Burden score if an excised sentinel lymph node was originally positive.

\section{Evaluation of the Surgical Specimen After Neoadjuvant Systemic Therapy}

Clinical information required for pathologic evaluation

It is important that the multidisciplinary team (e.g., surgeons, radiologists, and pathologists) communicate as a team for patient care; this is covered in detail in our companion multi-disciplinary paper. ${ }^{41}$ At a bare minimum, the request form must clearly indicate neoadjuvant systemic therapy has been given, along with the location and pretreatment size of the tumor(s). A suggested template requisition form that can be sent with the specimen is included in Supplementary Information 2.

\section{Specimen handling}

Priorities for evaluation of the surgical specimen are different after neoadjuvant systemic therapy, with emphasis on informed and accurate evaluation of tumor response to treatment. In general, one should apply the principles within national and institutional guidelines for standardization of processing and reporting of breast specimens, such as those noted above. Ideally, specimens should be sliced when fresh to identify the markers of the original tumor bed and to ensure formalin penetration.

Residual tumor is usually less well defined and softer than untreated tumor, making it more difficult to detect grossly. Therefore, careful mapping and more extensive sampling is required for histopathologic study. It is strongly recommended that an image of the sliced specimen be recorded (radiograph, photograph, photocopy, or drawing) and then used as a map for the sections taken, so that the histopathologic findings of any residual disease in the breast can be more easily understood. For example, the sections taken can be drawn on a printed image of the sliced specimen and then scanned into the pathology database for viewing at the time of histopathologic study. More precise imaging of the gross specimen and correlation with the histopathologic sections will decrease the number of sections taken from the breast, and increase the efficiency and accuracy of pathologic assessment. This can save time and money while enabling consistent and careful pathologic interpretation. The recommendations below will attempt to supplement existing national guidelines for specific situations encountered in the neoadjuvant setting; however, the pathologist should use sound clinical judgment on a case-by-case basis.

Sampling of small lumpectomy specimens. Many institutional standard operating procedures call for thinly slicing and submitting small specimens in their entirety (e.g., $<5 \mathrm{~cm}$ in greatest diameter in Yale University's standard operating procedure, and $<30$ g in the Dutch national guideline ${ }^{26}$ ) in a manner that allows reconstruction of the specimen at the time of microscopic evaluation through accurate description or with the help of a diagram. Unfortunately, this approach does not allow for tissue collection for research. Clinical judgment should be applied in this setting. If there is obvious gross residual tumor, then a research sample can be taken without compromising accurate histological assessment. In cases where the macroscopic findings are nonspecific, or there is clinical doubt about the location of the tumor bed, then consideration should be given to submitting the entire specimen. Research samples may still be taken by thinning the blocks and submitting the trim, or, alternatively, small cylinders of tissue can be taken with a punch biopsy tool. 


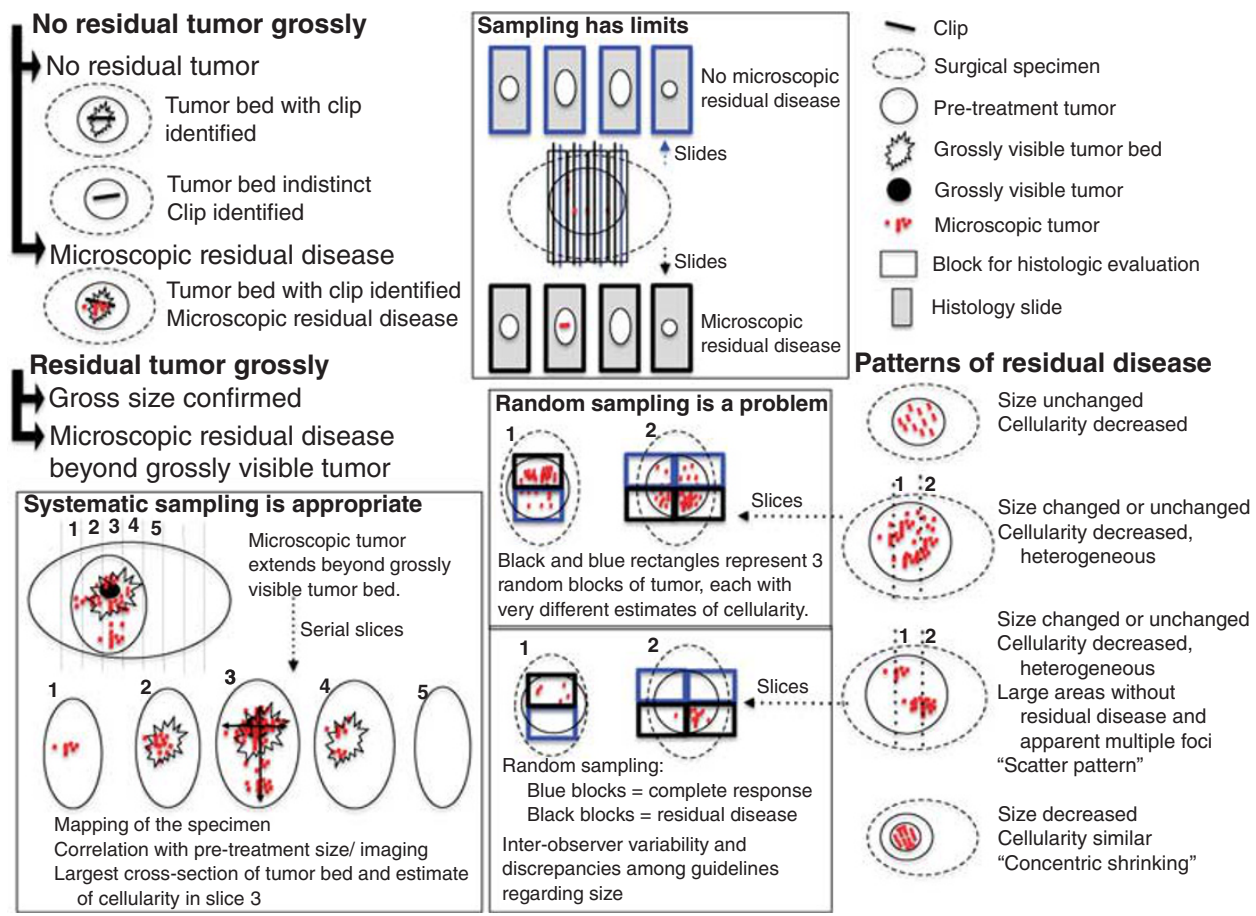

Figure 3 Problems related to sampling for histologic evaluation. Gross residual tumor may or may not be present after neoadjuvant therapy (top left). Even when the tumor bed is entirely submitted, histologic evaluation has limits (top center). The blue and black slides represent different levels obtained from the same block. The blue slides show a complete response. The black slides show minimal residual microscopic disease. Partial response shows various patterns and the decrease in cellularity is often heterogeneous (right). In these cases, random sampling of tumor can lead to very different estimates of tumor cellularity (bottom center). Random sampling with the blue blocks would conclude a complete response. Random sampling with the black blocks would document residual disease. Often, the microscopic tumor extends beyond a grossly visible tumor bed (bottom left). The largest cross-section of tumor bed is sampled for an estimate of tumor cellularity.

Depending on the type of processing used for the research tissue, histology can still be evaluated if deemed clinically necessary, such as hematoxylin and eosin-stained sections of research blocks. A previous international working group has addressed the collection of research tissue in the neoadjuvant setting in detail. ${ }^{43}$

It is important to document that these small resections have adequately excised the lesion. The tumor bed/clip must be identified. Tumor bed extending to the margins should be documented.

Sampling of large lumpectomy/mastectomy specimens (partial submission). Targeted representative sections can be taken from larger specimens, but it is essential to carefully and accurately represent the tumor bed in a manner that can be retrospectively mapped to the gross and/or radiologic findings. This enables more accurate estimation of the extent of residual disease. Correlation with clinical and imaging findings is imperative to ensure the correct area is sampled. Sampling should include grossly visible tumor bed and/or the location of any marker clips and immediately adjacent tissue to encompass the area suspected of involvement by carcinoma before treatment (Figure 3). This area to be sampled is referred to as the pretreatment area of involvement in the discussion below. Degree of sampling is then determined by the pretreatment size in addition to any visible tumor bed or grossly visible residual disease.

Ideally, the specimen is sliced to reveal the largest cross-section of the pretreatment area of involvement. Block(s) representing the full face of the pretreatment area of involvement should be taken of every $1 \mathrm{~cm}$ slice containing pretreatment area of involvement, or, for very large tumors, five representative blocks of a cross-section of pretreatment area of involvement per 1-2 cm of pretreatment size, up to a total maximum of $\sim 25$ blocks. In the absence of trial-based evidence as to the degree of sampling required, the committee felt this to be a pragmatic approach that should be sufficient to determine the presence of pathological complete response. The US Food and Drug Administration, in their guidance, have recommended taking 'a minimum of one block per $\mathrm{cm}$ of pre-treatment tumor size, or at least 10 blocks in total, whichever is greater' ${ }^{34}$ The extent of sampling should be guided by good clinical judgment on a case-by-case basis-informed, directed sampling is more important than blindly taking a prescribed number of blocks. For assessment of cellularity of very large tumor beds, five representative blocks are sufficient to represent the largest 
cross-section of residual tumor bed and calculate the Residual Cancer Burden. ${ }^{45}$

Precise description must be used to allow reconstruction of the specimen during histologic evaluation for accurate measurements and cellularity estimates. We strongly recommend visual images, such as photographs, specimen radiographs, or sketched diagrams, with annotations to indicate the sites where tissue sections were taken for histopathologic evaluation.

If no residual disease is seen on initial sections, or if the distribution of the disease does not correspond to the initial gross impression, then a second pass may be needed to submit further blocks. Additional blocks, including sections documenting margins, should be obtained as with non-neoadjuvant specimens.

Laboratories with access to large tissue cassettes are encouraged to utilize this technique as a superior method for mapping the residual tumor bed. Large cassettes enable sampling of a bigger area with fewer blocks, with the entire lesion often captured on a single slide. This simplifies reconstruction of the extent of residual disease, measurement of lesion size, and examination of margins. ${ }^{46}$

In cases where the above cutoffs would not result in submission of the entire tumor bed, remaining tissue can be sampled for research. Areas with grossly visible tumor can easily be sampled. Cases where the above cutoffs result in submission of the entire tumor bed can be sampled for research as described in the section 'Sampling of small lumpectomy specimens' under 'Specimen handling' above. If only formalin-fixed, paraffin-embedded tissue is needed, additional blocks can be submitted from a second pass for research from areas that had residual tumor on microscopy.

Multiple lesions in lumpectomy or mastectomy. In specimens containing multiple lesions, each lesion should be handled as a single lesion as described under 'Sampling of large lumpectomy/mastectomy specimens (partial submission)' above, with the addition of blocks of tissue taken from in between the lesions to ensure that they are truly separate and to evaluate the presence of other intervening disease, such as DCIS.

\section{Microscopic reporting}

Prognostic and predictive factors traditionally evaluated in surgical specimens following primary surgery are all relevant in the neoadjuvant systemic therapy setting. Although some familiar prognostic information may be altered by treatment (e.g., tumor grade and histological type) or may be less reliable (lymph node and margin status), much can be gained from the opportunity to evaluate response to treatment.

Histologic tumor type and grade. The method for determination of histologic tumor type and tumor grade is identical to that used for non-neoadjuvant specimens, although it is not clear whether these add prognostic information to the pretreatment results. Tumors with a typical appearance of no special type before treatment may have a lobular growth pattern following neoadjuvant chemotherapy. ${ }^{47}$ Treatment can cause nuclear hyperchromasia and pleomorphism; however, the findings should be compared with the pretreatment biopsy before assuming they are treatment-related. The mitotic rate may be reduced by treatment; this finding is associated with a better prognosis (disease-free survival and overall

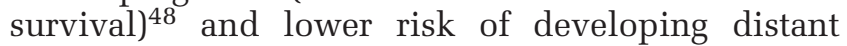
metastases. ${ }^{49}$ Clonal heterogeneity within the tumor may be reflected by variable response to therapy, and by areas with different morphology and grade. A comment regarding the presence of such heterogeneity should be made in the report, and is important when choosing blocks for postneoadjuvant systemic therapy hormone receptor and HER2 assessment.

If multiple, morphologically distinct tumors are present that are clearly separated by adipose tissue, they should be reported as separate lesions. However, it should be noted that the largest residual primary tumor is used for determination of both Residual Cancer Burden and yp stage. Note that ypT stage is defined by the largest contiguous focus of invasive cancer, whereas Residual Cancer Burden uses the two dimensions of the largest residual area of residual invasive cancer (i.e., that does not need to be contiguous) in the tumor bed.

Size and extent. Tumor size/extent is often more difficult to assess after neoadjuvant systemic therapy. There are two main patterns of tumor response following neoadjuvant systemic therapy-concentric shrinking and the scatter pattern (Figure 3). Measurement of lesion size in this latter scenario may be difficult. Our suggested approach is described in Table 1.

Cellularity. In addition to its effect on tumor size, neoadjuvant systemic therapy often has a profound effect on tumor cellularity. Tumor size may not decrease, but overall cellularity may be markedly reduced (Figure 3), making residual tumor cellularity an important factor in assessing response. ${ }^{50} \mathrm{Com}-$ parison of pre- and post-treatment cellularity is the key element of several systems for grading response. ${ }^{7,13,15,42}$ If a formal classification system for grading of response is used, this should be noted in the report. As tumor cellularity is often heterogeneous, the pretreatment core biopsy may not be representative of the entire tumor. Similarly, changes in tumor cellularity induced by neoadjuvant systemic therapy can be heterogeneous and therefore more extensive sampling may be needed to accurately assess cellularity. The descriptions of these scoring systems do not explicitly state how to deal with this heterogeneity, and it can be tempting only to assess the most cellular areas of the tumor. 
Table 1 Controversial scenarios in reporting breast cancer after neoadjuvant systemic therapy

Scenario Current evidence/guidelines Suggested approach

Residual tumor present as scattered foci (common)
Presence of lymphovascular invasion in the absence of an identifiable residual invasive tumor mass (rare)
- Tumor size is often more difficult to assess after neoadjuvant treatment.

- Residual carcinoma may be present as multiple, small foci scattered over a (ill-defined) tumor bed.

- There are two main options to measure size in this setting:

(A) Residual tumor bed size in two dimensions (used to calculate the Residual Cancer Burden score): the extent of the area involved by all islands of residual invasive tumor cells and intervening stroma. This does not include tumor bed beyond the area containing residual invasive tumor cells.

(B) Tumor size according to American Joint Committee on Cancer staging 7 th edition ${ }^{67}$ : if the residual tumor consists of microscopic nests in a fibrotic stroma, ypT should be based on the largest contiguous area of invasive carcinoma, with an indication that multiple foci are present ('m').

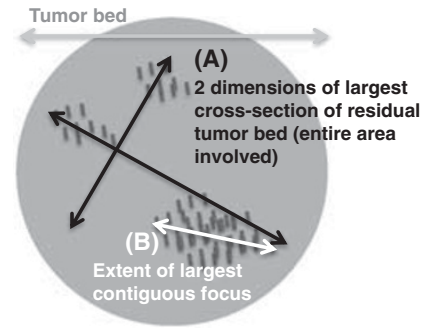

- There are insufficient data on the independent prognostic significance of the presence of lymphovascular invasion or extensive lymphovascular invasion in neoadjuvant specimens.

- One small study found that such intralymphatic tumor carries adverse prognostic significance, even in the absence of residual stromal invasion..$^{70}$ However, most of the patients also had residual disease in the lymph nodes and multivariate analysis was not possible.
Presence of isolated tumor cells in lymph nodes (common)
- American Joint Committee on Cancer TNM recommends isolated tumor cells after chemotherapy be called node negative $\left(\mathrm{ypNO}_{\mathrm{itc}}\right)$ but not regarded as pathological complete response. ${ }^{67}$
- If there is a single lesion present on pretreatment imaging, then regard residual disease as a single tumor, especially if tumor cells are present within a reactive stromal background consistent with a solitary tumor bed (Opinion).

- When there are scattered islands of tumor cells, measurement (B) (diagram at left) as described by American Joint Committee on Cancer staging 7 th edition ${ }^{67}$ may result in significant underestimation of tumor extent. There are also currently no data on the relationship of measurement (B) to outcome (Opinion).

- Lesion size including the cell clusters and intervening fibrous tissue (A) (diagram at left), which is congruent with the earlier, 6th edition of American Joint Committee on Cancer staging, ${ }^{68}$ correlates with survival ${ }^{69}$ and may be more relevant, for example for comparison with radiology (Limited Evidence).

- In our opinion, the combination of residual tumor cellularity and measurement (A) is the better indicator of response (Opinion).

- When required to report American Joint Committee on Cancer 7th edition stage, both measurements (A) and (B) should be given in the pathology report, with an explanation of how the final size and stage designation was made (Published Guideline).

- If there are multiple tumors present on pretreatment imaging or tumor foci are separated by normal breast tissue, then regard as multiple lesions and measure independently as distinct tumor foci. Dimensions from the largest tumor deposit should be used for ypTNM staging (Published Guideline).

- Residual lymphovascular invasion should NOT be classed as pathological complete response-make a statement in the pathology report that residual tumor is present in the form of intravascular disease (Opinion).

- Ensure tumor bed has been accurately localized and adequately sampled to exclude residual invasive disease (Opinion).

- Ensure truly lymphovascular invasion, not DCIS or retraction artifact. This may be difficult; immunohistochemistry may be helpful (Opinion).

- Measurement is optional. If a limited area is involved, a measurement in $\mathrm{mm}$ can be given. Alternatively, lymphovascular invasion can be quantified as focal or extensive with 'extensive' defined as one or more foci in more than one block ${ }^{71}$ (Opinion).

- Although it was agreed residual lymphovascular invasion should not be regarded as pathological complete response, in the absence of adequate data the working group felt it was not appropriate to give definite reporting recommendations (Opinion).

- Any residual disease in the lymph node, including micrometastases and isolated tumor cells, should NOT be classified as pathological complete response (Limited Evidence). 
Table 1 (Continued)

\begin{tabular}{|c|c|}
\hline Scenario & Current evidence/guidelines \\
\hline & $\begin{array}{l}\text { - World Health Organization recommends } \\
\text { isolated tumor cells after chemotherapy be } \\
\text { called node positive. }{ }^{72} \\
\text { - Findings include: } \\
\text { - Disease-free survival and overall survival } \\
\text { worsened with increasing number of nodes } \\
\text { and deposit size. Size of largest metastasis } \\
\text { was strongest predictor of overall survival in } \\
\text { multivariate analysis. Micrometastatic } \\
\text { disease }<2 \text { mm, including isolated tumor } \\
\text { cells, was predictive of worse outcome. }{ }^{40} \\
\text { - No difference in relapse-free survival and } \\
\text { overall survival between groups when size of } \\
\text { the largest residual metastatic deposit was } \\
\text { classified as } \leq 0.1 \text { cm, } 0.1-1 \text { cm, and } \geq 1 \mathrm{~cm} \\
\text { in patients with proven axillary nodal } \\
\text { disease before neoadjuvant chemotherapy. } \\
\text { - No change in prognosis with occult } \\
\text { metastases identified by } \\
\text { immunohistochemical staining for } \\
\text { cytokeratins. }{ }^{73}\end{array}$ \\
\hline
\end{tabular}

Suggested approach

- If no associated fibrosis, treat as in adjuvant setting and call node negative (Opinion).

- If associated fibrosis present, the likelihood is this represents previous micro- or macrometastasis with response. A comment should be included regarding the presence of chemotherapy effect, and the size of the entire area, including tumor cells and intervening stroma, should be measured, rather than the size of the largest cell cluster (Limited Evidence).

- Additional levels and/or immunohistochemistry are not routinely required. However, immunohistochemistry may be useful if suspicious cells are identified on hematoxylin and eosin, and levels can be used to clarify the size of a deposit if isolated tumor cells/micrometastasis are present on the initial section (Limited Evidence).

Limited Evidence $=$ consensus opinion of committee based on limited evidence; Opinion = consensus opinion of committee in the absence of reliable evidence.

The Residual Cancer Burden system does not require pretreatment cellularity, but proposes standardized sampling of the specimen with assessment of the average cellularity across the largest twodimensional area of residual tumor bed. For Residual Cancer Burden, the tumor bed area is defined by the two largest dimensions of gross tumor bed defined by macroscopic examination with or without accompanying specimen radiography, but can be later revised after these corresponding slides have been reviewed under the microscope. Hence, the importance of accurate block description and advisability of an illustrative map to determine how the slides map to the gross tumor bed (described above). The online cellularity standard provided in the Residual Cancer Burden website ${ }^{45}$ and the images in the publication for the Miller-Payne score are useful aids for pathologists in estimating cellularity. ${ }^{15}$ The presence or absence of residual DCIS, and the percentage of residual tumor present as in situ disease, should also be documented as per the Residual Cancer Burden.

We advocate submitting the largest cross-section of the residual tumor bed with the relevant sections noted in the pathology report.

Lymphovascular invasion. The presence or absence of lymphovascular invasion should be documented (Figure 4). There are insufficient data on the independent prognostic significance of lymphovascular invasion in neoadjuvant specimens. See Table 1 for suggested approaches to assessing and reporting lymphovascular invasion.

Margins. In cases with variable response leading to multiple, small foci of residual disease in a subtle

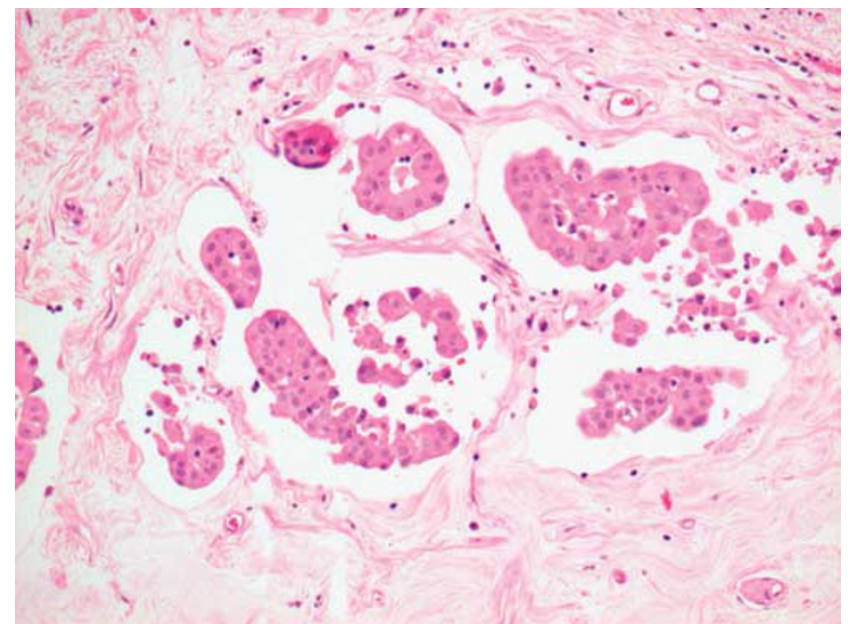

Figure 4 Extensive lymphovascular space invasion after chemotherapy. In this case, an invasive tumor focus was not identified despite extensive sampling. The axillary nodes were positive for residual metastatic carcinoma (courtesy of Elena Provenzano).

tumor bed, carcinoma may extend beyond an apparently negative margin. Tumor bed extending to the margins, and which margin is involved, should be documented (Figure 5).

\section{Evaluation of the axilla after treatment}

Several studies have shown that posttreatment nodal status is an important determinant of disease-free survival and overall survival, regardless of response within the breast. $^{32,35-40}$ Currently, lymph node staging in patients who have received neoadjuvant systemic therapy is usually performed by either 
sentinel lymph node biopsy or axillary lymph node dissection. The accuracy of sentinel lymph node biopsy for staging postneoadjuvant systemic therapy is still under investigation, especially in patients with clinically positive nodes before treatment. ${ }^{44,51}$ The paradigm in surgical management of the axilla is evolving, ${ }^{34}$ and is the subject of ongoing investigation. ${ }^{44,51}$ This is reflected in the use of the phrase 'sampled regional lymph nodes' by the US Food and Drug Administration in its proposed definition of pathological complete response. ${ }^{34}$

The procedure for evaluating sentinel lymph nodes and axillary lymph nodes should be the same as for non-neoadjuvant specimens. All surgically removed lymph nodes should be sectioned at $2 \mathrm{~mm}$ intervals and entirely submitted for histologic evaluation. Some special considerations apply, however.

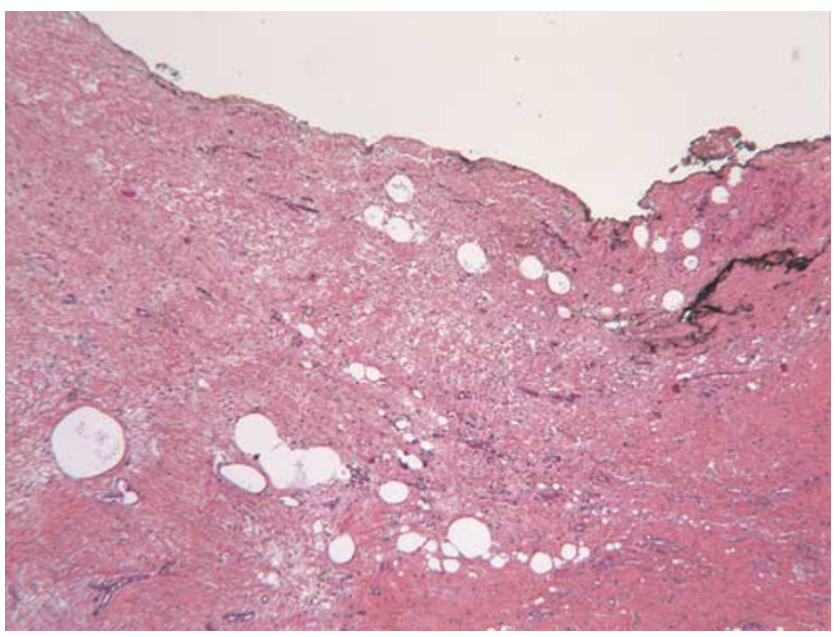

Figure 5 Tumor bed present at the resection margin (courtesy of Frédérique Penault-Llorca).
Some studies have indicated a lower number of lymph nodes identified at axillary lymph node dissection after neoadjuvant systemic therapy, whereas others have found no significant difference following careful pathological evaluation. ${ }^{52-54}$ Pathologists evaluating axillary lymph node dissection tissue should subject any tissue that may represent lymph node for microscopic evaluation.

The size of the largest metastatic deposit should be measured microscopically and the presence or absence of any extranodal extension documented. Postneoadjuvant systemic therapy tumor cells are often present as scattered single cells within an area of reactive stromal changes or lymphoid tissue. When measuring the size of the metastasis in this context, the size of the area that is even partly involved by metastatic tumor should be measured, and not just the size of the largest tumor cluster. Clearly separate smaller foci in a node are not included in the maximum size measurement. As micrometastases and isolated tumor cells found after neoadjuvant systemic therapy are predictors of worse survival, specimens with nodal micrometastases or isolated tumor cells should not be designated as having pathological complete response. ${ }^{40,55}$ Our suggested approach to assessing isolated tumor cells in this context is provided in Table 1.

The presence of treatment effect in the lymph nodes in the form of fibrosis (Figure 6), mucin pools, or large aggregates of foamy histiocytes identifies a subset of patients with an outcome intermediate between that of completely node negative and node positive after neoadjuvant systemic therapy. ${ }^{56} \mathrm{How}$ ever, small fibrous scars in lymph nodes can also be seen in patients without treatment, and in patients who have had a previous biopsy it can be impossible to reliably distinguish biopsy site changes from regressed metastasis. ${ }^{57}$ Previously involved nodes
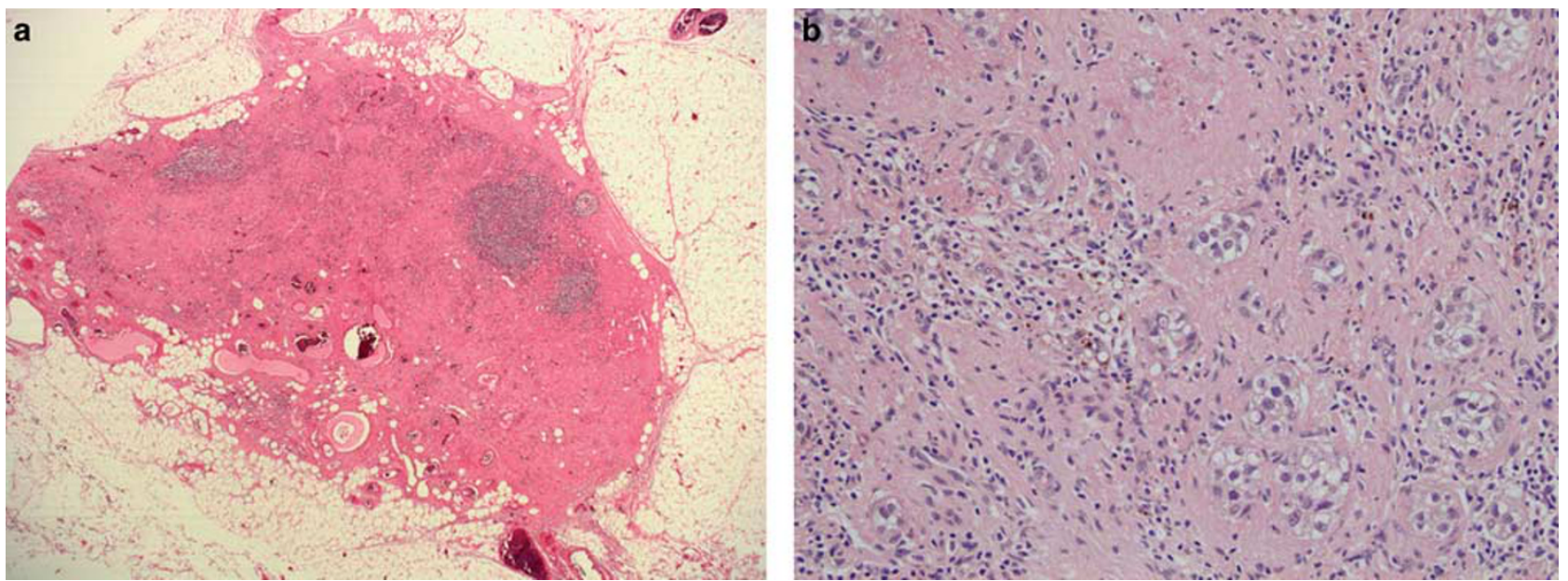

Figure 6 Lymph node showing zonal areas of fibrosis after chemotherapy indicative of metastasis with response to therapy (courtesy of Elena Provenzano). (a) Low-power image of lymph node showing zonal fibrosis indicating site of metastasis. (b) On higher magnification of a different node, residual islands of tumor cells are present in a setting of reactive fibrosis with hemosiderin-laden macrophages, consistent with chemotherapy effect. 
may also look completely normal after treatment. The latter scenario can cause concern when there was histologically proven metastasis before treatment, but evidence of a positive node cannot be found in the final surgical specimen. In this setting, the specimen (including axillary tail, if a mastectomy) should be carefully reexamined to ensure all nodes have been retrieved, and the patient reexamined, before assuming there has been complete response. Clipping the involved node before treatment can also be of value in determining nodal response.

In some centers, sentinel lymph nodes are assessed by molecular assays (e.g., one-step nucleic acid amplification) without any morphological evaluation. This does not allow assessment of response in the node; moreover, one-step nucleic acid amplification is usually not calibrated to detect isolated tumor cells. ${ }^{58}$ Therefore, we do not recommend the use of these techniques in the neoadjuvant setting.

\section{Pathological complete response}

Our group agrees with the following core principle of the definition of pathological complete response as proposed by the US Food and Drug Administration: 'Pathological complete response is defined as the absence of residual invasive cancer on.... evaluation of the complete resected breast specimen and all sampled regional lymph nodes following completion of neoadjuvant systemic therapy.' 34 However, we advocate that the presence of invasive tumor cells is considered residual disease regardless of the method of detection-that is, hematoxylin and eosin or immunohistochemistry-although the latter is not routinely recommended. The alternative definition, requiring absence of both DCIS and invasive carcinoma in the breast, can also be used. The definition of pathological complete response chosen should be agreed between pathologists and clinicians within individual institutions, and clearly stated in the report. If the patient is enrolled in a clinical trial, the definition of pathological complete response prescribed by the trial standard operating procedure should be included as part of the report with an explanatory note. Regardless of which definition is used, the presence/absence and extent of residual DCIS should be reported as detailed in our recommended template (Table 2).

Microscopically, the tumor bed may be identified as a focal area of loose, edematous reactive stroma with a variable inflammatory cell infiltrate that may include collections of lipid or hemosiderin-laden macrophages, lymphocytes, and plasma cells. Background breast lobules often appear hyalinized and atrophic with a perilobular lymphocytic infiltrate.

We would like to stress the following. Accurate, reproducible documentation of pathological complete response requires adequate sampling of the correct area of the breast. Overly exhaustive sampling and histologic evaluation of the entire tumor bed are generally not required and are far less valuable than intelligent mapping of the correct locations within the specimen. Therefore, correlation of clinical and imaging information and markers of the tumor site with gross pathology of the specimen are indispensible.

\section{Retesting of markers in the postneoadjuvant therapy specimen}

Reassessment of hormone receptor and HER2 status in residual cancer after neoadjuvant systemic therapy is variable between individual centers, with no consensus regarding if and when retesting of markers is advisable. The clinical utility of reassessing marker status in the surgical specimen may depend on the results from the core biopsies taken before neoadjuvant systemic therapy. If retesting is performed, it may be done on either the residual primary tumor or residual nodal disease if the latter contains a better representation of residual tumor cells. Our recommendations are provided in Table 3.

Finally, in some centers, assessment of Ki67 labeling index is performed before and after neoadjuvant systemic therapy. Posttreatment Ki67 index has been shown to correlate with long-term outcome after both neoadjuvant endocrine ${ }^{59}$ and chemotherapy, ${ }^{60,61}$ although its routine use in clinical practice has not yet been formally recommended because of lack of standardization in its assessment. ${ }^{62-64}$ Proliferation is commonly reduced by neoadjuvant systemic therapy and hence, in addition to Ki67, results of multigene assays that include proliferation genes may also change if assessed before and after treatment. ${ }^{65}$

\section{Minimum data set to be reported by pathologists}

A suggested summary template for reporting neoadjuvant systemic therapy specimens is presented in Table 2, with minimum data set items highlighted. The US National Cancer Institute's Breast Oncology Local Disease (BOLD) Task Force has also recommended standardized data elements for collection in preoperative breast cancer clinical trials. ${ }^{66}$

\section{Conclusion}

Postneoadjuvant systemic therapy histopathological changes are complex, and careful systematic review of the specimen is required for accurate diagnosis and follow-up treatment. For pathological complete response to be used as an indicator of response to novel therapies, it is essential to have a standardized way in which residual disease is measured and reported. We designed the recommendations specifically for the clinical trial setting; however, they can be optionally incorporated into routine practice because, in our opinion, standardization is most effective when uniformly applied. Hopefully, such standardization will improve our knowledge and ability to compare outcomes, promote the submission of specimens for translational research, 
Table 2 Suggested template for reporting breast cancer specimens after neoadjuvant systemic therapy in clinical trials ${ }^{\mathrm{a}}$

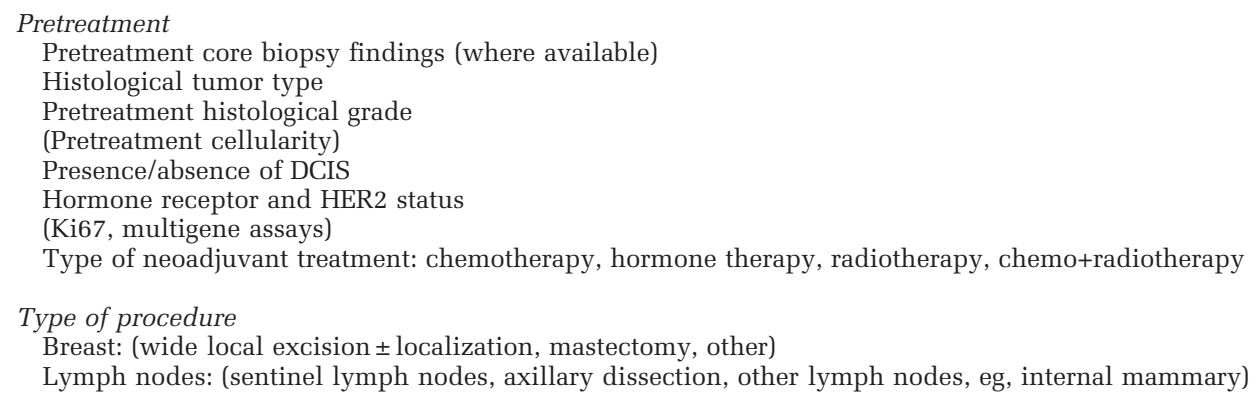

Laterality: (left, right, not specified)

Macroscopy

Residual macroscopic tumor identified: yes/ no

If residual macroscopic tumor:

Site of tumor (upper outer, lower outer, upper inner, lower inner, central)

Unifocal vs multifocal

If multifocal, number of foci

Size of macroscopic lesion(s): $\mathrm{x}_{-} \mathrm{x}_{-} \mathrm{mm}$

If no residual macroscopic tumor:

Area of fibrosis present: yes/ no

Site of fibrosis

Size of fibrosis: $\mathrm{x}_{-} \mathrm{x}_{-} \mathrm{mm}$

Radiological marker identified: yes/no/not present

Microscopy

Size/extent of residual tumor: _ mm

Largest cross-section of residual tumor bed (entire area involved) $\mathrm{x}_{-} \mathrm{mm}$ represented in cassettes (....)

Posttreatment histological grade

Residual cellularity:_\%

DCIS: present/absent

Total lesion size including DCIS:_ $\mathrm{x}_{-} \mathrm{mm}$

Percentage of residual cellularity that is CIS:_\%

Lymphovascular space invasion: present/absent/indeterminate/extensive

In the absence of residual tumor, is the previous tumor site identified (clip site/area of fibrosis): yes/no

Margin status

Invasive carcinoma: present/absent; distance to closest margin

DCIS: present/absent; distance to closest margin

Tumor bed: present/absent

Lymph node status

Number of sentinel/axillary lymph nodes

Number of sentinel/axillary lymph nodes with metastases

Size of largest metastasis

Evidence of treatment response in the metastases: present/absent

Number of lymph nodes with evidence of treatment response (e.g., fibrosis or histiocytic infiltrate) but no tumor cells

Presence (extent) of extracapsular extension

Final classification of chemotherapy response

Grade of response and classification system used

If no formal grading system used, minimum comment regarding response as below:

Breast:

Pathological Complete Response

Residual invasive carcinoma, no definite response

Residual invasive carcinoma with probable or definite response to chemotherapy

*If there is more than one tumor with variable response between lesions, then the poorest level of response should be taken as the overall classification.

Lymph nodes:

Metastasis present, no response

Metastasis present, evidence of response

No residual metastasis but evidence of previous metastasis with response

No metastasis or fibrosis (true negative)

ypTN stage

Repeat marker testing:

ER/PR/HER2 if initial biopsy was negative or equivocal

(Ki67)

Abbreviations: CIS, carcinoma in situ; DCIS, ductal carcinoma in situ; ER, estrogen receptor; PR, progesterone receptor.

${ }^{a}$ Bold items indicate suggested minimum data set specific to postneoadjuvant specimens, IN ADDITION to minimum required for other types of specimens. 
Table 3 Retesting of hormone receptors and HER2 after neoadjuvant therapy

\begin{tabular}{|c|c|c|}
\hline Recommendation & Clinical setting & Comments \\
\hline $\begin{array}{l}\text { - Routine reassessment not } \\
\text { currently recommended }\end{array}$ & $\begin{array}{l}\text { - Positive ER/PR/HER2 result on } \\
\text { pretreatment core biopsy }\end{array}$ & $\begin{array}{l}\text { - No change in marker expression in the majority of } \\
\text { patients. } \\
\text { - Uncertainty whether conversion should inform the } \\
\text { choice of future adjuvant therapies (i.e., to stop or start a } \\
\text { targeted treatment). }{ }^{7-76} \\
\text { - Unknown independent prognostic value of marker } \\
\text { conversion for disease-free and overall survival. } \\
\text { However, loss of HER2 amplification following } \\
\text { neoadjuvant trastuzumab has been associated with worse } \\
\text { outcome. }{ }^{77}\end{array}$ \\
\hline $\begin{array}{l}\text { - Reassessment must be } \\
\text { performed }\end{array}$ & $\begin{array}{l}\text { - Retesting of markers is required } \\
\text { as part of a clinical trial protocol } \\
\text { - Biomarker status not known }\end{array}$ & $\begin{array}{l}\text { - Reassessment of ER/PR and HER2 in residual invasive } \\
\text { disease should be included in clinical trial protocols to } \\
\text { gather high-quality data to clarify the significance of } \\
\text { change of receptor status on outcome. }\end{array}$ \\
\hline $\begin{array}{l}\text { - Reassessment should be } \\
\text { considered }\end{array}$ & $\begin{array}{l}\text { - Negative or equivocal result on } \\
\text { pretreatment core biopsy } \\
\text { - Insufficient invasive tumor for } \\
\text { accurate assessment or DCIS only } \\
\text { on pretreatment core biopsy } \\
\text { - Retesting requested by clinicians } \\
\text { - Biopsy performed in another } \\
\text { institution } \\
\text { - Heterogeneous tumor or multiple } \\
\text { tumors with different morphologies } \\
\text { on resection } \\
\text { - No response to therapy }\end{array}$ & $\begin{array}{l}\text { - Two different meta-analyses of published studies have } \\
\text { reported a mean prevalence of discordant results pre- and } \\
\text { post-neoadjuvant therapy of } 13 \text { and } 18 \% \text { for ER, } 32 \text { and } \\
26 \% \text { for PR, and } 9 \text { and } 6 \% \text { for HER } 2.78,79 \\
\text { - Causes of discordance include technical artifacts, } \\
\text { misinterpretation of test results, intratumoral } \\
\text { heterogeneity of marker expression, and changes induced } \\
\text { by the intervening therapies }{ }^{80} \text { (eg, inclusion of } \\
\text { trastuzumab in neoadjuvant treatment may increase the } \\
\text { rate of negative conversion for HER2).77,81 } \\
\text { - Retesting may give a positive result in a small percentage } \\
\text { of patients. } \\
\text { - Positive result would indicate eligibility for targeted } \\
\text { therapy. } \\
\text { - If HER } 2 \text { status reassessed and found to be discordant, } \\
\text { retesting should be performed with both } \\
\text { immunohistochemistry and in situ hybridization. }\end{array}$ \\
\hline
\end{tabular}

Abbreviations: DCIS, ductal carcinoma in situ; ER, estrogen receptor; PR, progesterone receptor.

${ }^{a}$ Pretreatment core biopsy should be adequate for unequivocal diagnosis of invasive carcinoma and assessment of key prognostic and predictive markers as this forms the only tumor sample if there is a complete pathological response. If this is not the case, repeat core biopsy should be performed or primary surgery considered.

and facilitate the more timely introduction of new agents.

The recommendation of this committee is that pathologic reports of residual disease after neoadjuvant chemotherapy and/or targeted therapy in clinical trials should include the following information:

- Pathological Complete Response or Residual Disease. This should separately describe whether there was residual invasive cancer in the breast, in situ cancer in the breast, and the pathologic status of the regional lymph nodes.

- Residual Cancer Burden as the preferred method for more detailed quantification of residual disease. The report should provide the final residual tumor dimensions, cellularity of cancer in the final tumor bed area and the proportion of in situ component within that cancer, and the number of positive nodes and the size of the largest metastasis, as well as the Residual Cancer Burden score and class.

- ypTN Stage. The report should separately report the ypT and ypN stages and the pathologist should use the most current edition of the American Joint Committee on Cancer/Union for International
Cancer Control staging definitions when evaluating tumor size after neoadjuvant chemotherapy.

\section{Acknowledgments}

We thank the BIG-NABCG leadership: Dr Nancy E Davidson, MD, of the University of Pittsburgh Cancer Institute and UPMC Cancer Center, Pittsburgh, PA; Dr Martine Piccart, MD, PhD, of Institut Jules Bordet, Université Libre de Bruxelles, Brussels, Belgium; Dr Larry Norton, MD, of the Memorial Sloan-Kettering Cancer Center, New York, NY. We also thank the following for providing their input: Dr Helena Earl of the University of Cambridge Department of Oncology, United Kingdom, Chief Investigator of Neo-tAnGo and ARTemis trials; Dr Keith Amos of University of North Carolina, Chapel Hill; Dr Véronique Becette of Institut Curie/Hôpital René Huguenin, France; Dr Wojciech Biernat of the Medical University of Gdansk, Poland; Dr Hervé Bonnefoi of Institut Bergonié, France; $\mathrm{Dr}$ Aman Buzdar of $\mathrm{MD}$ Anderson Cancer Center, Texas; Dr Vicente Peg Cámara of Vall d'Hebron University Hospital, Barcelona, Spain; Dr Paul Cane and Dr Sarah Pinder of Guy's and St Thomas' Hospitals, 
London; Dr Lesley Carson of Aberdeen Royal Infirmary, Foresterhill, NHS Grampian, Aberdeen, United Kingdom; Dr Diana Dickson-Witmer of Christiana Care, Delaware; Dr Gyungyub Gong of Asan Medical Center, University of Ulsan College of Medicine, Korea; Dr Jimmy Green of Pathology Sciences Medical Group, Norfolk, Virginia; Dr Chih-Yi Hsu and Dr Ling-Ming Tseng of Taipei Veterans General Hospital, Taipei, Taiwan; Dr Judith Kroep of Leiden University Medical Center, The Netherlands; Dr A Marilyn Leitch and Dr Venetia Sarode of UT Southwestern Medical Center, Texas; Dr Eleftherios Mamounas of the National Surgical Adjuvant Breast and Bowel Project; Dr Paul Kelly Marcom of Duke University, North Carolina; Dr Paolo Nuciforo of Vall d'Hebron Institute of Oncology, Barcelona, Spain; Dr Soonmyung Paik of the Yonsei University College of Medicine, Seoul, Korea, and the National Surgical Adjuvant Breast and Bowel Project; Dr David Peston of Charing Cross Hospital, London; Dr Jean-Yves Pierga of Institut Curie, France; Dr Roberto Salgado of Institut Jules Bordet, Belgium; Dr Miguel Quintela-Fandino of Centro Nacional de Investigaciones Oncológicas, Spain; Dr William Sikov of Women and Infants Hospital, Breast Health Center, Rhode Island; Drs Emilio Alba, Ana Lluch, and Joan Albanell of GEICAM (Spanish Breast Cancer Research Group), Spain; Dr Jeremy Thomas of Western General Hospital, NHS Lothian, Edinburgh; Dr Gary Unzeitig of Laredo Breast Care, Texas; Dr Jelle Wesseling of Netherlands Cancer Institute; and Dr Marc Wilt of Centre Paul Strauss, Strasbourg, France. We thank Rebecca Enos of the Emmes Corporation for information gathering and for coordination and administrative support of the BIG-NABCG Residual Disease Characterization Working Group. We thank the Breast Cancer Research Foundation for its support of the BIG-NABCG collaboration, including the BIG-NABCG meeting where this working group was proposed.

\section{Disclosure/conflict of interest}

Dr Symmans filed Residual Cancer Burden as intellectual property (Nuvera Biosciences), patenting the Residual Cancer Burden equation. (The Residual Cancer Burden equation is freely available on the worldwide web.) Dr Symmans reports current stock in Nuvera Biosciences and past stock in Amgen.

\section{References}

1 Esserman LJ, Woodcock J. Accelerating identification and regulatory approval of investigational cancer drugs. JAMA 2011;306:2608-2609.

2 Cortazar P, Zhang L, Untch $\mathrm{M}$ et al. Pathological complete response and long-term clinical benefit in breast cancer: the CTNeoBC pooled analysis. Lancet 2014;384:164-172.
3 Mamounas EP, Anderson SJ, Dignam JJ et al. Predictors of locoregional recurrence after neoadjuvant chemotherapy: results from combined analysis of National Surgical Adjuvant Breast and Bowel Project B-18 and B-27. J Clin Oncol 2012;30:3960-3966.

4 Apple SK, Suthar F. How do we measure a residual tumor size in histopathology (the gold standard) after neoadjuvant chemotherapy? Breast 2006;15: 370-376.

5 Kuroi K, Toi M, Tsuda $\mathrm{H}$ et al. Issues in the assessment of the pathologic effect of primary systemic therapy for breast cancer. Breast Cancer 2006;13:38-48.

6 Marchio C, Sapino A. The pathologic complete response open question in primary therapy. J Natl Cancer Inst Monogr 2011;2011:86-90.

7 Pinder SE, Provenzano E, Earl $\mathrm{H}$ et al. Laboratory handling and histology reporting of breast specimens from patients who have received neoadjuvant chemotherapy. Histopathology 2007;50:409-417.

8 Sahoo S, Lester SC. Pathology of breast carcinomas after neoadjuvant chemotherapy: an overview with recommendations on specimen processing and reporting. Arch Pathol Lab Med 2009;133:633-642.

9 Fan F. Evaluation and reporting of breast cancer after neoadjuvant chemotherapy. Open Pathol J 2009;3: 58-63.

10 Symmans WF, Peintinger F, Hatzis C et al. Measurement of residual breast cancer burden to predict survival after neoadjuvant chemotherapy. J Clin Oncol 2007;25:4414-4422.

11 Chollet P, Abrial C, Durando X et al. A new prognostic classification after primary chemotherapy for breast cancer: residual disease in breast and nodes (RDBN). Cancer J 2008;14:128-132.

12 Chollet P, Amat S, Belembaogo E et al. Is Nottingham prognostic index useful after induction chemotherapy in operable breast cancer? Br J Cancer 2003;89: 1185-1191.

13 Sataloff DM, Mason BA, Prestipino AJ et al. Pathologic response to induction chemotherapy in locally advanced carcinoma of the breast: a determinant of outcome. J Am Coll Surg 1995;180:297-306.

14 Penault-Llorca F, Abrial C, Raoelfils I et al. Comparison of the prognostic significance of Chevallier and Sataloff's pathologic classifications after neoadjuvant chemotherapy of operable breast cancer. Hum Pathol 2008;39:1221-1228.

15 Ogston KN, Miller ID, Payne S et al. A new histological grading system to assess response of breast cancers to primary chemotherapy: prognostic significance and survival. Breast 2003;12:320-327.

16 Romero A, Garcia-Saenz JA, Fuentes-Ferrer $M$ et al. Correlation between response to neoadjuvant chemotherapy and survival in locally advanced breast cancer patients. Ann Oncol 2013;24:655-661.

17 Corben AD, Abi-Raad R, Popa I et al. Pathologic response and long-term follow-up in breast cancer patients treated with neoadjuvant chemotherapy: a comparison between classifications and their practical application. Arch Pathol Lab Med 2013;137:1074-1082.

18 Peintinger F, Sinn B, Hatzis C et al. Reproducibility of residual cancer burden for prognostic assessment of breast cancer after neoadjuvant chemotherapy. Mod Pathol 2015;28:913-920.

19 Symmans WF, Wei C, Gould R et al. Long-term prognostic value of residual cancer burden (RCB) classification following neoadjuvant chemotherapy 
[abstract S6-02]. San Antonio Breast Cancer Symposium: San Antonio, TX, USA, 2013.

20 Sheri A, Smith IE, Johnston SR et al. Residual proliferative cancer burden to predict long-term outcome following neoadjuvant chemotherapy. Ann Oncol 2015;26:75-80.

21 Mittendorf EA, Jeruss JS, Tucker SL et al. Validation of a novel staging system for disease-specific survival in patients with breast cancer treated with neoadjuvant chemotherapy. J Clin Oncol 2011;29:1956-1962.

22 Royal College of Pathologists of Australasia (RCPA). Royal College of Pathologists of Australasia Invasive Breast Cancer Structured Reporting Protocol. 2nd edn. Version 2.0. November 2012.

23 Lambein K, Van de Vijver K, Faverly D et al. Belgian guidelines for laboratory handling and pathology reporting of breast carcinoma after neoadjuvant therapy. Belg J. Med Oncol 2011;5:144-153.

24 Arbeitsgemeinschaft Gynaekologische Onkologie Studiengruppe (AGO) http://www.ago-online.de/de/fuermediziner/leitlinien/mamma/en/ Accessed 30 October 2014.

25 NHS Breast Screening Programme (NHSBSP). Pathology Reporting Of Breast Disease: A Joint Document Incorporating the Third Edition of the NHS Breast Screening Programme's Guidelines for Pathology Reporting in Breast Cancer Screening and the Second Edition of The Royal College of Pathologists' Minimum Dataset for Breast Cancer Histopathology. NHSBSP Publication No 58. NHS Cancer Screening Programmes jointly with The Royal College of Pathologists; 2005.

26 Integraal Kankercentrum Nederland. Beoordeling na neoadjuvante chemo- of endocriene therapie http:// www.oncoline.nl/breastcancer 2012. Accessed 30 October 2014.

27 College of American Pathologists. Protocol for the Examination of Specimens from Patients with Invasive Carcinoma of the Breast. June 2012 http://www.cap.org/apps/ docs/committees/cancer/cancer_protocols/2012/BreastIn vasive_12protocol_3100.pdf Accessed 30 October 2014.

28 Leyland-Jones BR, Ambrosone CB, Bartlett J et al. Recommendations for collection and handling of specimens from Group breast cancer clinical trials. J Clin Oncol 2008;26:5638-5644.

29 Provenzano E, Vallier AL, Champ R et al. A central review of histopathology reports after breast cancer neoadjuvant chemotherapy in the neo-tango trial. Br J Cancer 2013;108:866-872.

30 Chevallier B, Roche H, Olivier JP et al. Inflammatory breast cancer. Pilot study of intensive induction chemotherapy (FEC-HD) results in a high histologic response rate. Am J Clin Oncol 1993;16:223-228.

31 Dieras V, Fumoleau P, Romieu G et al. Randomized parallel study of doxorubicin plus paclitaxel and doxorubicin plus cyclophosphamide as neoadjuvant treatment of patients with breast cancer. J Clin Oncol 2004;22:4958-4965.

32 von Minckwitz G, Untch M, Blohmer JU et al. Definition and impact of pathologic complete response on prognosis after neoadjuvant chemotherapy in various intrinsic breast cancer subtypes. J Clin Oncol 2012;30: 1796-1804.

33 Mazouni C, Peintinger F, Wan-Kau S et al. Residual ductal carcinoma in situ in patients with complete eradication of invasive breast cancer after neoadjuvant chemotherapy does not adversely affect patient outcome. J Clin Oncol 2007;25:2650-2655.
34 U.S. Food and Drug Administration. Guidance for Industry: Pathological Complete Response in Neoadjuvant Treatment of High-Risk Early-Stage Breast Cancer: Use as an Endpoint to Support Accelerated Approval. October 2014 http://www.fda.gov/downloads/Drugs/ GuidanceComplianceRegulatoryInformation/Gui dances/UCM305501.pdf Accessed 30 October 2014.

35 Rouzier R, Extra JM, Klijanienko J et al. Incidence and prognostic significance of complete axillary downstaging after primary chemotherapy in breast cancer patients with T1 to T3 tumors and cytologically proven axillary metastatic lymph nodes. J Clin Oncol 2002;20: 1304-1310.

36 McCready DR, Hortobagyi GN, Kau SW et al. The prognostic significance of lymph node metastases after preoperative chemotherapy for locally advanced breast cancer. Arch Surg 1989;124:21-25.

37 Rastogi P, Anderson SJ, Bear HD et al. Preoperative chemotherapy: updates of National Surgical Adjuvant Breast and Bowel Project Protocols B-18 and B-27. J Clin Oncol 2008;26:778-785.

38 Buchholz TA, Tucker SL, Masullo L et al. Predictors of local-regional recurrence after neoadjuvant chemotherapy and mastectomy without radiation. J Clin Oncol 2002;20:17-23.

39 Hennessy BT, Hortobagyi GN, Rouzier R et al. Outcome after pathologic complete eradication of cytologically proven breast cancer axillary node metastases following primary chemotherapy. J Clin Oncol 2005;23: 9304-9311.

40 Klauber-DeMore N, Ollila DW, Moore DT et al. Size of residual lymph node metastasis after neoadjuvant chemotherapy in locally advanced breast cancer atients is prognostic. Ann Surg Oncol 2006;13: 685-691.

41 Bossuyt V, Provenzano E, Symmans WF et al. Recommendations for standardized pathological characterization of residual disease for neoadjuvant clinical trials of breast cancer by the BIG-NABCG collaboration. Ann Oncol 2015;26:1280-1291.

42 Sinn HP, Schmid H, Junkermann H et al. [Histologic regression of breast cancer after primary (neoadjuvant) chemotherapy]. Geburtshilfe und Frauenheilkunde 1994;54:552-558.

43 Loi S, Symmans WF, Bartlett JM et al. Proposals for uniform collection of biospecimens from neoadjuvant breast cancer clinical trials: timing and specimen types. Lancet Oncol 2011;12:1162-1168.

44 Kuehn T, Bauerfeind I, Fehm T et al. Sentinel-lymphnode biopsy in patients with breast cancer before and after neoadjuvant chemotherapy (SENTINA): a prospective, multicentre cohort study. Lancet Oncol 2013;14:609-618.

45 Residual Cancer Burden calculator and associated documents [Guide for Measuring Cancer Cellularity, Examples of Gross \& Microscopic Evaluation, Pathology Protocol for Macroscopic and Microscopic Assessment of RCB] http://www3.mdanderson.org/app/ medcalc/index.cfm? pagename $=$ jsconvert 3 Accessed 30 October 2014.

46 Ibarra JA. The value of combined large format histopathology technique to assess the surgically removed breast tissue following neoadjuvant chemotherapy: a single institution study of 40 cases. Int J Breast Cancer 2012;2012:361707.

47 Carder P. Typing breast cancer following primary chemotherapy. Histopathology 1999;35:584-585. 
48 Penault-Llorca F, Abrial C, Raoelfils I et al. Changes and predictive and prognostic value of the mitotic index, Ki-67, cyclin D1, and cyclo-oxygenase-2 in 710 operable breast cancer patients treated with neoadjuvant chemotherapy. Oncologist 2008;13:1235-1245.

49 Diaz J, Stead L, Shapiro N et al. Mitotic counts in breast cancer after neoadjuvant systemic chemotherapy and development of metastatic disease. Breast Cancer Res Treat 2013;138:91-97.

50 Rajan R, Poniecka A, Smith TL et al. Change in tumor cellularity of breast carcinoma after neoadjuvant chemotherapy as a variable in the pathologic assessment of response. Cancer 2004;100:1365-1373.

51 Boughey JC, Suman VJ, Mittendorf EA et al. Sentinel lymph node surgery after neoadjuvant chemotherapy in patients with node-positive breast cancer: the ACOSOG Z1071 (Alliance) clinical trial. JAMA 2013;310:1455-1461.

52 Belanger J, Soucy G, Sideris L et al. Neoadjuvant chemotherapy in invasive breast cancer results in a lower axillary lymph node count. J Am Coll Surg 2008;206:704-708.

53 Neuman H, Carey LA, Ollila DW et al. Axillary lymph node count is lower after neoadjuvant chemotherapy. Am J Surg 2006;191:827-829.

54 Boughey JC, Donohue JH, Jakub JW et al. Number of lymph nodes identified at axillary dissection: effect of neoadjuvant chemotherapy and other factors. Cancer 2010;116:3322-3329.

55 Fisher ER, Wang J, Bryant J et al. Pathobiology of preoperative chemotherapy: findings from the National Surgical Adjuvant Breast and Bowel (NSABP) protocol B-18. Cancer 2002;95:681-695.

56 Newman LA, Pernick NL, Adsay V et al. Histopathologic evidence of tumor regression in the axillary lymph nodes of patients treated with preoperative chemotherapy correlates with breast cancer outcome. Ann Surg Oncol 2003;10:734-739.

57 Donnelly J, Parham DM, Hickish $\mathrm{T}$ et al. Axillary lymph node scarring and the association with tumour response following neoadjuvant chemoendocrine therapy for breast cancer. Breast 2001;10:61-66.

58 Feldman S, Krishnamurthy S, Gillanders W et al. A novel automated assay for the rapid identification of metastatic breast carcinoma in sentinel lymph nodes. Cancer 2011;117:2599-2607.

59 Ellis MJ, Tao Y, Luo J et al. Outcome prediction for estrogen receptor-positive breast cancer based on postneoadjuvant endocrine therapy tumor characteristics. J Natl Cancer Inst 2008;100:1380-1388.

60 Jones RL, Salter J, A'Hern R et al. The prognostic significance of Ki67 before and after neoadjuvant chemotherapy in breast cancer. Breast Cancer Res Treat 2009;116:53-68.

61 von Minckwitz G, Schmitt W, Loibl S et al. Ki67 measured after neoadjuvant chemotherapy for primary breast cancer. Clin Cancer Res 2013;19: 4521-4531.

62 Harris L, Fritsche H, Mennel R et al. American Society of Clinical Oncology 2007 update of recommendations for the use of tumor markers in breast cancer. J Clin Oncol 2007;25:5287-5312.

63 Polley MC, Leung S, McShane LM et al. An International Ki67 Reproducibility Study. J Natl Cancer Inst 2013;105:1897-1906.

64 Dowsett M, Nielsen TO, A'Hern R et al. Assessment of Ki67 in breast cancer: recommendations from the
International Ki67 in Breast Cancer working group. J Natl Cancer Inst 2011;103:1656-1664.

65 Earl HM, Chin S, Dunning M et al. Neo-tAnGo science: a translational study of PAM 50 sub-typing in sequential fresh tissue samples during neoadjuvant chemotherapy [abstract \#1015]. J Clin Oncol 2013;31, (Suppl; abstr 1015).

66 National Cancer Institute. Breast Oncology Local Disease (BOLD) Task Force of the Breast Cancer Steering Committee. NCI BOLD Task Force Common Data Elements (CDEs) http://www.cancer.gov/aboutnci/ organization/ccct/steering-committees/breast-cancer/ Accessed 30 October 2014.

67 Edge SB, Byrd DR, Compton CC et al. (eds) American Joint Committee on Cancer (AJCC) Cancer Staging Manual. 7th edn. Springer-Verlag: New York, 2009.

68 Greene FL, Page DL, Fleming ID et al. (eds) American Joint Committee on Cancer (AJCC) Cancer Staging Manual. 6th edn. Springer-Verlag: New York, 2002.

69 Carey LA, Metzger R, Dees EC et al. American Joint Committee on Cancer tumor-node-metastasis stage after neoadjuvant chemotherapy and breast cancer outcome. J Natl Cancer Inst 2005;97:1137-1142.

70 Rabban JT, Glidden D, Kwan ML et al. Pure and predominantly pure intralymphatic breast carcinoma after neoadjuvant chemotherapy: an unusual and adverse pattern of residual disease. Am J Surg Pathol 2009;33:256-263.

71 Colleoni M, Rotmensz N, Maisonneuve P et al. Prognostic role of the extent of peritumoral vascular invasion in operable breast cancer. Ann Oncol 2007;18: 1632-1640.

72 Lakhani SR, Ellis IO, Schnitt SJ et al. WHO Classification of Tumours of the Breast. 4th edn. World Health Organization, 2012.

73 Loya A, Guray M, Hennessy BT et al. Prognostic significance of occult axillary lymph node metastases after chemotherapy-induced pathologic complete response of cytologically proven axillary lymph node metastases from breast cancer. Cancer 2009;115: 1605-1612.

74 Chen S, Chen CM, Yu KD et al. Prognostic value of a positive-to-negative change in hormone receptor status after neoadjuvant chemotherapy in patients with hormone receptor-positive breast cancer. Ann Surg Oncol 2012;19:3002-3011.

75 Hirata T, Shimizu C, Yonemori K et al. Change in the hormone receptor status following administration of neoadjuvant chemotherapy and its impact on the longterm outcome in patients with primary breast cancer. Br J Cancer 2009;101:1529-1536.

76 Tacca O, Penault-Llorca F, Abrial C et al. Changes in and prognostic value of hormone receptor status in a series of operable breast cancer patients treated with neoadjuvant chemotherapy. Oncologist 2007;12:636-643.

77 Mittendorf EA, Wu Y, Scaltriti M et al. Loss of HER2 amplification following trastuzumab-based neoadjuvant systemic therapy and survival outcomes. Clin Cancer Res 2009;15:7381-7388.

78 Jabbour MN, Massad CY, Boulos FI. Variability in hormone and growth factor receptor expression in primary versus recurrent, metastatic, and postneoadjuvant breast carcinoma. Breast Cancer Res Treat 2012;135:29-37.

79 Zhang N, Moran MS, Huo Q et al. The hormonal receptor status in breast cancer can be altered by neoadjuvant chemotherapy: a meta-analysis. Cancer Invest 2011;29:594-598. 
80 Valent A, Penault-Llorca F, Cayre A et al. Change in HER2 (ERBB2) gene status after taxane-based chemotherapy for breast cancer: polyploidization can lead to diagnostic pitfalls with potential impact for clinical management. Cancer Genet 2013;206: $37-41$.

Supplementary Information accompanies the paper on Modern Pathology website (http://www.nature.com/ modpathol)
81 von Minckwitz G, Darb-Esfahani S, Loibl S et al. Responsiveness of adjacent ductal carcinoma in situ and changes in HER2 status after neoadjuvant chemotherapy/trastuzumab treatment in early breast cancer-results from the GeparQuattro study (GBG 40). Breast Cancer Res Treat 2012;132:863-870. 